\title{
IDENTITIES OF GRADED SIMPLE ALGEBRAS
}

\author{
DUŠAN REPOVŠ AND MIKHAIL ZAICEV
}

\begin{abstract}
We study identities of finite dimensional algebras over a field of characteristic zero, graded by an arbitrary groupoid $\Gamma$. First we prove that its graded colength has a polynomially bounded growth. For any graded simple algebra $A$ we prove the existence of the graded PI-exponent, provided that $\Gamma$ is a commutative semigroup. If $A$ is simple in a non-graded sense the existence of the graded PI-exponent is proved without any restrictions on $\Gamma$.
\end{abstract}

\section{INTRODUCTION}

We study numerical characteristics of identities of finite dimensional graded simple algebras over a field of characteristics zero. The main object of our investigations is the asymptotic behaviour of sequences of graded codimensions and graded colengths of such algebras (all necessary definitions and notions will be given in the next section). Given a graded algebra $A$, one can associate the sequence of socalled graded codimensions $\left\{c_{n}^{g r}(A), n=1,2, \ldots\right\}$. This sequence is an important numerical invariant of graded identities of $A$. It is known that this sequence is exponentially bounded, that is $c_{n}^{g r}(A) \leq a^{n}$ for some real $a$, provided that $\operatorname{dim} A<\infty$. In this case the following natural question arises: does the limit

$$
\exp ^{g r}(A)=\lim _{n \rightarrow \infty} \sqrt[n]{c_{n}^{g r}(A)}
$$

exist and what are its possible values? If the limit (1) exists then it is called the graded PI-exponent of $A$.

In the non-graded case, codimension growth is well understood. Existence and integrality of the (non-graded) PI-exponent was conjectured by Amitsur in 1980's for associative PI-algebras. Amitsur's conjecture was confirmed in [1, 2]. Later the same result was proved for finite dimensional Lie algebras $[3,4,5]$, Jordan and alternative algebras $[6,7,8]$ and many other algebraic systems. In the general nonassociative case, for any real $\alpha>1$, examples of algebras with PI-exponent equal to $\alpha$ were constructed in [9]. Recently, the first example of algebra $A$ such that the PI-exponent of $A$ does not exist, was constructed [10]. Nevertheless, for any finite dimensional simple algebra the PI-exponent does exist [11].

For graded algebras there are only partial results of this kind. For example, if $A$ is an associative graded PI-algebra then its graded PI-exponent always exists and it is an integer [12]. An existence of the $\mathbb{Z}_{2}$-graded PI-exponent for any finite dimensional simple Lie superalgebra has recently been proved in [13]. Note that for finite dimensional Lie superalgebras, both graded and ordinary PI-exponents can be fractional $[11,14,15]$. The main purpose of this paper is to prove the existence of

2010 Mathematics Subject Classification. Primary 17B01, 16P90; Secondary 16R10.

Key words and phrases. Polynomial identities, graded algebras, codimensions, exponential growth. 
graded PI-exponents for any finite dimensional graded simple algebra (see Theorem 2).

Another important numerical characteristic of identities of an algebra $A$ is the socalled colength sequence $l_{n}(A)$. Except for its independent interest, asymptotic behaviour of $\left\{l_{n}(A)\right\}$ plays an important role in the studies of asymptotics of $\left\{c_{n}(A)\right\}$. The polynomial type of growth of $\left\{l_{n}(A)\right\}$ is very convenient for investigations of codimension growth.

Polynomial upper bounds of the colength for any associative PI-algebra were established in [16]. For an arbitrary (non-associative) finite dimensional algebra the same restriction was obtained in [17]. In the case of finite dimensional Lie superalgebras, polynomial growth of $\mathbb{Z}_{2}$-graded colength has recently been confirmed in [18]. In order to get the main result of the paper we will find the polynomial upper bound for graded colength of a finite dimensional graded algebra (see Theorem 1).

\section{Preliminaries}

Let $\Gamma$ be a groupoid. An $F$-algebra $A$ is said to be $\Gamma$-graded if there is a vector space decomposition

$$
A=\bigoplus_{g \in \Gamma} A_{g}
$$

and $A_{g} A_{h} \subseteq A_{g h}$ for all $g, h \in \Gamma$. An element $a \in A$ is called homogeneous of degree $g$ if $a \in A_{g}$ and in this case we write $\operatorname{deg}_{\Gamma} a=g$. A subspace $V \subseteq A$ is homogeneous iff $V=\bigoplus_{g \in \Gamma}\left(V \cap A_{g}\right)$. We call $A$ graded simple if it has no homogeneous ideals. For instance, if $\Gamma$ is a group and $A=F[\Gamma]$ is its group algebra then $A$ is $\Gamma$-graded simple but is not simple in the usual sense. On the other hand, any simple algebra with an arbitrary grading is graded simple.

We recall some key notions from the theory of graded and ordinary identities and their numerical invariants. We refer the reader to $[19,20]$ for details. Consider an absolutely free algebra $F\{X\}$ with a free generating set

$$
X=\bigcup_{g \in \Gamma} X_{g}, \quad\left|X_{g}\right|=\infty \text { for any } g \in \Gamma .
$$

One can define a $\Gamma$-grading on $F\{X\}$ by setting $\operatorname{deg}_{\Gamma} x=g$, when $x \in X_{g}$, and extend this grading to the entire $F\{X\}$ in the natural way. A polynomial $f\left(x_{1}, \ldots, x_{n}\right)$ in homogeneous variables $x_{1} \in X_{g_{1}}, \ldots, x_{n} \in X_{g_{n}}$ is called a graded identity of a $\Gamma$-graded algebra $A$ if $f\left(a_{1}, \ldots, a_{n}\right)=0$ for any $a_{1} \in A_{g_{1}}, \ldots, a_{n} \in A_{g_{n}}$. The set $I d^{g r}(A)$ of all graded identities of $A$ forms an ideal of $F\{X\}$ which is stable under graded homomorphisms $F\{X\} \rightarrow F\{X\}$.

First, let $\Gamma$ be finite, $\Gamma=\left\{g_{1}, \ldots, g_{t}\right\}$ and $X=X_{g_{1}} \cup \ldots \cup X_{g_{t}}$. Denote by $P_{n_{1}, \ldots, n_{t}}$ the subspace of $F\{X\}$ of multilinear polynomials of total degree $n=$ $n_{1}+\cdots+n_{t}$ in variables $x_{1}^{(1)}, \ldots, x_{n_{1}}^{(1)} \in X_{g_{1}}, \ldots, x_{1}^{(t)}, \ldots, x_{n_{t}}^{(t)} \in X_{g_{t}}$. Then the value

$$
c_{n_{1}, \ldots, n_{t}}(A)=\operatorname{dim} \frac{P_{n_{1}, \ldots, n_{t}}}{P_{n_{1}, \ldots, n_{t}} \cap I^{g r}(A)}
$$

is called a partial codimension of $A$ while

$$
c_{n}^{g r}(A)=\sum_{n_{1}+\cdots+n_{t}=n}\left(\begin{array}{c}
n \\
n_{1}, \ldots, n_{t}
\end{array}\right) c_{n_{1}, \ldots, n_{t}}(A)
$$


is called a graded codimension of $A$. Recall that the support of the grading is the set

$$
\text { Supp } A=\left\{g \in \Gamma \mid A_{g} \neq 0\right\} .
$$

Note that if Supp $A \neq \Gamma$, say, Supp $A=\left\{g_{1}, \ldots, g_{k}\right\}, k<t$, then the value

$$
\sum_{n_{1}+\cdots+n_{k}=n}\left(\begin{array}{c}
n \\
n_{1}, \ldots, n_{k}
\end{array}\right) \operatorname{dim} \frac{P_{n_{1}, \ldots, n_{k}}}{P_{n_{1}, \ldots, n_{k}} \cap I d^{g r}(A)}
$$

coincides with (2). This allows us to consider (3) as the definition of the graded codimension of $A$ even if $\Gamma$ is infinite, provided that Supp $A=\left\{g_{1}, \ldots, g_{k}\right\}$.

For convenience, denote

$$
P_{n_{1}, \ldots, n_{k}}(A)=\frac{P_{n_{1}, \ldots, n_{k}}}{P_{n_{1}, \ldots, n_{k}} \cap \operatorname{Id} d^{g r}(A)} .
$$

Given $1 \leq j \leq k$, consider the action of the symmetric group $S_{n_{j}}$ on $P_{n_{1}, \ldots, n_{k}}$ defined by

$$
\sigma f\left(\ldots, x_{1}^{(j)}, \ldots, x_{n_{j}}^{(j)}, \ldots\right)=f\left(\ldots, x_{\sigma(1)}^{(j)}, \ldots, x_{\sigma\left(n_{j}\right)}^{(j)}, \ldots\right) .
$$

Then the spaces $P_{n_{1}, \ldots, n_{k}}$ and $P_{n_{1}, \ldots, n_{k}}(A)$ become $F[H]$-modules, where $H=S_{n_{1}} \times$ $\cdots \times S_{n_{k}}$. Any $F[H]$-module $P_{n_{1}, \ldots, n_{k}}(A)$ is decomposed into the sum of irreducible $F[H]$-submodules and in the languages of group characters it can be written as

$$
\chi_{H}\left(P_{n_{1}, \ldots, n_{k}}(A)\right)=\sum_{\lambda^{(1)} \vdash n_{1}, \ldots, \lambda^{(k)} \vdash n_{k}} m_{\lambda^{(1)}, \ldots, \lambda^{(k)}} \chi_{\lambda^{(1)}, \ldots, \lambda(k)} .
$$

Here, $\chi_{\lambda^{(1)} \ldots \ldots \lambda^{(k)}}$ is the character of the irreducible $H$-representation defined by the $k$-tuple $\left(\lambda^{(1)}, \ldots, \lambda^{(k)}\right)$ of partitions $\lambda^{(1)} \vdash n_{1}, \ldots, \lambda^{(k)} \vdash n_{k}$ and $m_{\lambda^{(1)}} \ldots, \lambda^{(k)}$ is the multiplicity of the corresponding $F[H]$-module in $P_{n_{1}, \ldots, n_{k}}(A)$. The integer

$$
l_{\lambda^{(1)}, \ldots, \lambda^{(k)}}(A)=\sum_{\lambda^{(1)} \vdash n_{1}, \ldots, \lambda^{(k)} \vdash n_{k}} m_{\lambda^{(1)}, \ldots, \lambda^{(k)}}
$$

is called the partial colength, whereas the integer

$$
l_{n}^{g r}(A)=\sum_{n_{1}+\cdots+n_{k}=n} l_{n_{1}, \ldots, n_{k}}(A)
$$

is called the graded colength of $A$.

As it was mentioned in the introduction, graded codimensions are exponentially bounded if $A$ is finite dimensional. Namely,

$$
c_{n}^{g r}(A) \leq d^{n+1}
$$

where $d=\operatorname{dim} A$ (see [21] and also [7, Proposition 2]). This result was proved in $[7,21]$ under the assumption that $\Gamma$ is a finite group. The same argument is valid for an arbitrary groupoid. Relation (8) allows us to consider upper and lower limits of $\sqrt[n]{c_{n}^{g r}(A)}$ and we can define the lower and the upper graded PI-exponents as follows:

$$
\underline{\exp }^{g r}(A)=\liminf _{n \rightarrow \infty} \sqrt[n]{c_{n}^{g r}(A)}, \quad \overline{\exp ^{g r}}(A)=\limsup _{n \rightarrow \infty} \sqrt[n]{c_{n}^{g r}(A)} .
$$

If the lower and the upper limits coincide then we also define the graded PI-exponent by

$$
\exp ^{g r}(A)=\underline{\exp }^{g r}(A)=\overline{\exp }^{g r}(A) .
$$


Representation theory of symmetric groups is a useful tool for studying asymptotics of codimension growth. Basic notions of $S_{n}$-representations can be found in [22] and its application to PI-theory in [19, 20].

Recall that, given a partition $\lambda \vdash n$, there is exactly one (up to isomorphism) irreducible $S_{n}$-representation defined by $\lambda$. Its character and dimension are denoted by $\chi_{\lambda}$ and $\chi_{\lambda}(1)=d_{\lambda}$, respectively. For the group $H=S_{n_{1}} \times \cdots \times S_{n_{k}}$ any irreducible representation is defined by the $k$-tuple of partitions $\lambda^{(1)} \vdash n_{1}, \ldots, \lambda^{(k)} \vdash$ $n_{k}$ and its character and dimension are $\chi_{\lambda(1), \ldots, \lambda(k)}$. Moreover,

$$
\chi_{\lambda^{(1), \ldots, \lambda}(k)}(1)=d_{\lambda^{(1)}} \cdots d_{\lambda^{(k)}},
$$

respectively. In particular, (5) and (9) imply the equality

$$
\begin{gathered}
c_{n_{1}, \ldots, n_{k}}(A)=\chi_{H}\left(P_{n_{1}, \ldots, n_{k}}(A)\right)(1)= \\
\sum_{\lambda^{(1)} \vdash n_{1}, \ldots, \lambda(k) \vdash n_{k}} m_{\lambda^{(1)}, \ldots, \lambda^{(k)}} d_{\lambda^{(1)}} \cdots d_{\lambda^{(k)}} .
\end{gathered}
$$

Let $d \geq 1$ be a fixed integer and let $\nu=\left(\nu_{1}, \ldots, \nu_{q}\right) \vdash m$ be a partition of $m$ with $q \leq d$. Dimension of an irreducible $F\left[S_{m}\right]$-module with the character $\chi_{\nu}$ is closely connected with the following function:

$$
\Phi(\nu)=\frac{1}{\left(\frac{\nu_{1}}{m}\right)^{\frac{\nu_{1}}{m}} \cdots\left(\frac{\nu_{d}}{m}\right)^{\frac{\nu_{d}}{m}}} .
$$

Here we assume that $\nu_{q+1}=\ldots=\nu_{d}=0$ in the case $q<d$ and $0^{0}=1$. The values $\Phi(\nu)^{m}$ and $d_{\nu}$ are close in the following sense.

Lemma 1. (see [11, Lemma 1]) Let $m \geq 100$. Then

$$
\frac{\Phi(\nu)^{m}}{m^{d^{2}+d}} \leq d_{\nu} \leq m \Phi(\nu)^{m} .
$$

We will use the following property of $\Phi$. Let $\nu$ and $\rho$ be any two partitions of $m$, such that $\nu=\left(\nu_{1}, \ldots, \nu_{p}\right), \rho=\left(\rho_{1}, \ldots, \rho_{q}\right), p, q \leq d$ and $q=p$ or $q=p+1, \rho_{p+1}=1$. As before, we consider $\rho$ and $\nu$ as partitions with $d$ components. We say that the Young diagram $D_{\rho}$ is obtained from diagram $D_{\nu}$ by pushing down one box if there exist $1 \leq i<j \leq d$ such that $\rho_{i}=\nu_{i}-1, \rho_{j}=\nu_{j}+1$ and $\rho_{t}=\nu_{t}$ for all remaining $1 \leq t \leq d$

Lemma 2. (see [11, Lemma 3], [23, Lemma 2]) Let $D_{\rho}$ be obtained from $D_{\nu}$ by pushing down one box. Then $\Phi(\rho) \geq \Phi(\nu)$.

\section{Polynomial Growth of GRAded Colength}

Consider a finite dimensional $\Gamma$-graded algebra $A$ with the support $\operatorname{Supp} A=$ $\left\{g_{1}, \ldots, g_{k}\right\}, A=A_{g_{1}} \oplus \cdots \oplus A_{g_{k}}$. Let

$$
d_{1}=\operatorname{dim} A_{g_{1}}, \ldots, d_{k}=\operatorname{dim} A_{g_{k}}
$$

be dimensions of the homogeneous components. Recall that an irreducible $F\left[S_{t}\right]$ module corresponding to the partition $\mu \vdash t$ can be realized as a minimal left $F\left[S_{t}\right]$ ideal generated by an essential idempotent $e_{T_{\lambda}}$ where $T_{\lambda}$ is some Young tableaux 
with Young diagram $D_{\lambda}$. For $H=S_{n_{1}} \times \cdots \times S_{n_{k}}$, any irreducible $F[H]$-module is isomorphic to the tensor product of $F\left[S_{n_{1}}\right], \ldots, F\left[S_{n_{k}}\right]$-modules with characters $\chi_{\lambda^{(1)}}, \ldots, \chi_{\lambda^{(k)}}$, respectively. The following remark easily follows from the construction of essential idempotents and therefore we omit the proof.

Lemma 3. Let $\lambda^{(1)}=\left(\lambda_{1}^{(1)}, \ldots, \lambda_{q_{1}}^{(1)}\right), \ldots, \lambda^{(k)}=\left(\lambda_{1}^{(k)}, \ldots, \lambda_{q_{k}}^{(k)}\right)$ be partitions of $n_{1}, \ldots, n_{k}$, respectively. Suppose that the multiplicity $m_{\lambda^{(1)}, \ldots, \lambda^{(k)}}$ on the right hand side of (5) is nonzero. Then $q_{1} \leq d_{1}, \ldots, q_{k} \leq d_{k}$.

For convenience we shall assume as before that $q_{1}=d_{1}, \ldots, q_{k}=d_{k}$ even if $q_{i}$ is strictly less than $d_{i}$ for some $i$.

Denote by $R=R\left\{X_{g_{1}} \cup \ldots \cup X_{g_{k}}\right\}$ the relatively free algebra of the variety var $A$ of $\Gamma$-graded algebras generated by $A$. Denote by $R_{d_{1}, \ldots, d_{k}}^{n_{1}, \ldots, n_{k}}$ the subspace of polynomials in $R$ of degree $n_{1}$ in the set of variables $\left\{X_{1}^{(1)}, \ldots, X_{d_{1}}^{(1)}\right\} \subseteq X_{g_{1}}$, of degree $n_{2}$ in $\left\{X_{1}^{(2)}, \ldots, X_{d_{2}}^{(2)}\right\} \subseteq X_{g_{2}}$, etc.

Lemma 4. Multiplicities on the right hand side of (5) satisfy the inequalities

$$
m_{\lambda^{(1)}, \ldots, \lambda(k)} \leq \operatorname{dim} R_{d_{1}, \ldots, d_{k}}^{n_{1}, \ldots, n_{k}} .
$$

Proof. Let $\widetilde{P}_{n_{1}, \ldots, n_{k}}$ be the subspace of multilinear polynomials of degree $n=$ $n_{1}+\cdots+n_{k}$ on $x_{1}^{(1)}, \ldots, x_{n_{1}}^{(1)}, \ldots, x_{1}^{(k)}, \ldots, x_{n_{k}}^{(k)}$ in $R$. Here $x_{j}^{(i)} \in X_{g_{i}}$ for all $1 \leq i \leq k, 1 \leq j \leq n_{i}$. Then $\widetilde{P}_{n_{1}, \ldots, n_{k}}$ is isomorphic to $P_{n_{1}, \ldots, n_{k}}(A)$ as an $F[H]$ module. Denote for brevity $q=m_{\lambda^{(1)}, \ldots, \lambda^{(k)}}$ and consider the $F[H]$-submodule

$$
M=M_{1} \oplus \cdots \oplus M_{q}
$$

of $\widetilde{P}_{n_{1}, \ldots, n_{k}}$, where $M_{1}, \ldots, M_{q}$ are isomorphic irreducible $F[H]$-modules with $H$ character $\chi_{\lambda^{(1)}, \ldots, \lambda(k)}$. Any $M_{j}$ is generated as an $F[H]$-module by a multilinear polynomial of the type

$$
\begin{gathered}
f_{j}\left(x_{1}^{(1)}, \ldots, x_{n_{1}}^{(1)}, \ldots, x_{1}^{(k)}, \ldots, x_{n_{k}}^{(k)}\right)= \\
e_{T_{\lambda^{(1)}}} \cdots e_{T_{\lambda^{(k)}}} h_{j}\left(x_{1}^{(1)}, \ldots, x_{n_{1}}^{(1)}, \ldots, x_{1}^{(k)}, \ldots, x_{n_{k}}^{(k)}\right)
\end{gathered}
$$

with a multilinear polynomial $h_{j} \in \widetilde{P}_{n_{1}, \ldots, n_{k}}$.

One can split the set of indeterminates $x_{1}^{(1)}, \ldots, x_{n_{k}}^{(k)}$ into a disjoint union of subsets

$$
\begin{aligned}
& P_{1}^{(1)}=\left\{x_{1}^{(1)}, \ldots, x_{\lambda_{1}^{(1)}}^{(1)}\right\}, \\
& P_{2}^{(1)}=\left\{x_{\lambda_{1}^{(1)}+1}^{(1)}, \ldots, x_{\lambda_{1}^{(1)}+\lambda_{2}^{(1)}}^{(1)}\right\}, \\
& P_{d_{1}}^{(1)}=\left\{x_{n_{1}-\lambda_{d_{1}}^{(1)}+1}^{(1)}, \ldots, x_{n_{1}}^{(1)}\right\}, \\
& P_{1}^{(k)}=\left\{x_{1}^{(k)}, \ldots, x_{\lambda_{1}^{(k)}}^{(k)}\right\}, \\
& P_{2}^{(k)}=\left\{x_{\lambda_{1}^{(k)}+1}^{(k)}, \ldots, x_{\lambda_{1}^{(k)}+\lambda_{2}^{(k)}}^{(k)}\right\},
\end{aligned}
$$




$$
P_{d_{k}}^{(k)}=\left\{x_{n_{k}-\lambda_{d_{k}}^{(1)}+1}^{(k)}, \ldots, x_{n_{k}}^{(k)}\right\}
$$

so that all $f_{1}, \ldots, f_{q}$ are symmetric on any subset $P_{1}^{(1)}, \ldots, P_{d_{k}}^{(k)}$.

Now we identify all variables in each symmetric subset, that is, we apply a homomorphism $\varphi: R \rightarrow R$ such that

$$
\begin{aligned}
& \text { - } \varphi\left(x_{\alpha}^{(j)}\right)=x_{1}^{(j)} \text { if } 1 \leq \alpha \leq \lambda_{1}^{(j)}, \\
& \quad \cdots \\
& \text { - } \varphi\left(x_{\alpha}^{(j)}\right)=x_{d_{j}}^{(j)} \text { if } \lambda_{1}^{(j)}+\cdots+\lambda_{d_{j}-1}^{(j)}<\alpha \leq n_{j}
\end{aligned}
$$

for all $j=1, \ldots, k$. Then all $\varphi\left(f_{1}\right), \ldots, \varphi\left(f_{q}\right)$ lie in $R_{d_{1}, \ldots, d_{k}}^{n_{1}, \ldots, n_{k}}$. Note that the total linearization of each of $\varphi\left(f_{j}\right)$ is equal to $f_{j}$ with a nonzero coefficient independent of $j$. Hence a nontrivial linear relation $\alpha_{1} \varphi\left(f_{1}\right)+\cdots+\alpha_{q} \varphi\left(f_{q}\right)=0$ implies the same relation $\alpha_{1} f_{1}+\cdots+\alpha_{q} f_{q}=0$. But $f_{1}, \ldots, f_{q}$ belong to distinct irreducible summands $M_{1}, \ldots, M_{q}$, respectively. In particular, they are linearly independent. Hence $q$ does not exceed $\operatorname{dim} R_{d_{1} \ldots, \ldots d_{k}}^{n_{1}, \ldots, n_{k}}$, and the proof is completed.

Now we restrict the dimension of $R_{d_{1}, \ldots, d_{k}}^{n_{1}, \ldots, n_{k}}$.

Lemma 5. Let $A=A_{g_{1}} \oplus \cdots \oplus A_{g_{k}}$ be a $\Gamma$-graded algebra with the support $\left\{g_{1}, \ldots, g_{k}\right\}$ and let $d_{1}=\operatorname{dim} A_{g_{1}}, \ldots, d_{k}=\operatorname{dim} A_{g_{k}}$. Then

$$
\operatorname{dim} R_{d_{1}, \ldots, d_{k}}^{n_{1}, \ldots, n_{k}} \leq\left(d_{1}+\cdots+d_{k}\right)\left(n_{1}+1\right)^{d_{1}^{2}} \cdots\left(n_{k}+1\right)^{d_{k}^{2}} .
$$

Proof. Let $\left\{a_{1}^{\left(g_{i}\right)}, \ldots, a_{d_{i}}^{\left(g_{i}\right)}\right\}$ be a basis of the subspace $A_{g_{i}}, 1 \leq i \leq k$. Consider a polynomial ring $F[Y]$, where $Y=Y_{g_{1}} \cup \ldots \cup Y_{g_{k}}$ and

$$
Y_{g_{i}}=\left\{y_{m, j}^{g_{i}} \mid 1 \leq m \leq d_{i}, j=1,2, \ldots\right\} .
$$

Then algebra $F[Y]$ can be naturally endowed by a $\Gamma$-grading with $\operatorname{Supp} F[Y]=$ $\left\{g_{1}, \ldots, g_{k}\right\}$ if we set $\operatorname{deg}_{\Gamma} y=g_{i}$ when $y \in Y_{g_{i}}$. Denote $\widetilde{A}=A \otimes F[Y]$ and fix elements

$$
z_{j}^{g_{i}}=\sum_{m=1}^{d_{i}} a_{m}^{\left(g_{i}\right)} \otimes y_{m, j}^{g_{i}}, \quad j=1,2, \ldots,
$$

in $\widetilde{A}$. Then $\operatorname{alg}\left\{z_{j}^{g_{i}}\right\}$ is also a $\Gamma$-graded algebra, where $\operatorname{deg} z_{j}^{g_{i}}=g_{i}$. Moreover, $\widetilde{A} \simeq$ $R$ and $R_{d_{1}, \ldots, d_{k}}^{n_{1}, \ldots, n_{k}}$ is a subspace of $A \otimes T$, where $T$ is the subspace of $F[Y]$ spanned by monomials of degree at most $n_{t}$ in the set of indeterminates $\left\{y_{m, j}^{g_{t}} \mid 1 \leq m, j \leq d_{t}\right\}$, $t=1, \ldots, k$. Clearly,

$$
\operatorname{dim} T \leq\left(n_{1}+1\right)^{d_{1}^{2}} \cdots\left(n_{k}+1\right)^{d_{k}^{2}}
$$

hence (11) follows from (12).

Now we are ready to get an upper bound for graded colength of $A$.

Theorem 1. Let $A=\bigoplus_{g \in \Gamma} A_{g}$ be a finite dimensional algebra graded by groupoid $\Gamma$ with Supp $A=\left\{g_{1}, \ldots, g_{k}\right\}$. Let also $\operatorname{dim} A_{g_{i}}=d_{i}, 1 \leq i \leq k$. Then the nth graded colength of $A$ satisfies the inequality

$$
l_{n}^{g r} \leq d(n+1)^{k+d_{1}^{2} \cdots+d_{k}^{2}+d_{1}+\cdots+d_{k}}
$$

where $d=\operatorname{dim} A=d_{1}+\cdots+d_{k}$. 
Proof. By Lemma 3, the total number of partitions $\lambda_{i} \vdash n_{i}$ does not exceed $\left(n_{i}+1\right)^{d_{i}}$ for any $i=1, \ldots, k$. Hence, by (6) and Lemmas 4 and 5 , we have

$$
l_{n_{1}, \ldots, n_{k}}(A) \leq d\left(n_{1}+1\right)^{d_{1}^{2}+d_{1}} \cdots\left(n_{k}+1\right)^{d_{k}^{2}+d_{k}}
$$

and

as follows from (7).

$$
l_{n}^{g r} \leq d(n+1)^{k+d_{1}^{2} \cdots+d_{k}^{2}+d_{1}+\cdots+d_{k}}
$$

\section{Existence of GRAded PI-EXPonents}

We begin this section with a technical result connecting dimensions of irreducible representations of symmetric groups and multinomial coefficients. Given a partition $\mu=\left(\mu_{1}, \ldots, \mu_{t}\right) \vdash m$, we denote by $q \mu\left(q \mu_{1}, \ldots, q \mu_{t}\right)$ the partition of $q m$, where $q \geq 1$ is an arbitrary integer. We also define the height $h t(\mu)$ as $h t(\mu)=t$. Recall that $d_{\mu}=\chi_{\mu(1)}$ is the dimension of the corresponding irreducible representation of $S_{m}$.

Lemma 6. Let $n_{1}, \ldots, n_{k}$ be positive integers, $n_{1}+\cdots+n_{k}=n \geq 100$. Let also $\lambda^{(1)}, \ldots, \lambda^{(k)}$ be partitions of $n_{1}, \ldots, n_{k}$, resp., such that $h t\left(\lambda^{(1)}\right), \ldots, h t\left(\lambda^{(k)}\right) \leq d$. If $q \geq 100$ then

$$
\left(\begin{array}{c}
q n \\
q n_{1}, \ldots, q n_{k}
\end{array}\right) d_{q \lambda^{(1)}} \cdots d_{q \lambda^{(k)}} \geq\left(\frac{1}{q n}\right)^{k\left(d^{2}+d+1\right)}\left[\frac{1}{n^{2 k}}\left(\begin{array}{c}
n \\
n_{1}, \ldots, n_{k}
\end{array}\right) d_{\lambda^{(1)}} \cdots d_{\lambda^{(k)}}\right]^{q} .
$$

Proof. Given nonnegative real $\alpha_{1}, \ldots \alpha_{k}$ with $\alpha_{1}+\cdots+\alpha_{k}=1$, we denote

$$
\Phi\left(\alpha_{1}, \ldots \alpha_{k}\right)=\frac{1}{\left(\alpha_{1}\right)^{\alpha_{1}} \cdots\left(\alpha_{k}\right)^{\alpha_{k}}} .
$$

From the Stirling formula for factorials it easily follows that

$$
\frac{1}{m^{k}} \Phi\left(\frac{m_{1}}{m}, \cdots, \frac{m_{k}}{m}\right)^{m} \leq\left(\begin{array}{c}
m \\
m_{1}, \ldots, m_{k}
\end{array}\right) \leq m \Phi\left(\frac{m_{1}}{m}, \cdots, \frac{m_{k}}{m}\right)^{m},
$$

where $m_{1}, \ldots, m_{k}$ are nonnegative integers and $m_{1}+\cdots+m_{k}=m$. Applying (13) to

we obtain

$$
P=\left(\begin{array}{c}
q n \\
q n_{1}, \ldots, q n_{k}
\end{array}\right)
$$

$$
P>\left(\frac{1}{q n}\right)^{k} \Phi\left(\frac{q n_{1}}{q n}, \ldots, \frac{q n_{k}}{q n}\right)^{q n}=\left(\frac{1}{q n}\right)^{k}\left[\Phi\left(\frac{n_{1}}{n}, \ldots, \frac{n_{k}}{n}\right)^{n}\right]^{q} .
$$

Applying again (13) we get

$$
P>\left(\frac{1}{q n}\right)^{k}\left[\frac{1}{n}\left(\begin{array}{c}
n \\
n_{1}, \ldots, n_{k}
\end{array}\right)\right]^{q} .
$$

It follows from Lemma 1 and (13) that

$$
\begin{aligned}
d_{q \lambda^{(1)}} \cdots d_{q \lambda^{(k)}}> & \left(\frac{1}{q n_{1}} \cdots \frac{1}{q n_{k}}\right)^{k\left(d^{2}+d\right)}\left[\Phi\left(\lambda^{(1)}\right)^{n_{1}} \cdots \Phi\left(\lambda^{(k)}\right)^{n_{k}}\right]^{q}> \\
& \left(\frac{1}{q n}\right)^{k\left(d^{2}+d\right)}\left[d_{\lambda^{(1)}} \cdots d_{\lambda^{(k)}}\right]^{q} .
\end{aligned}
$$


Now our lemma is a consequence of (14) and (15).

Recall that $A$ is a $d$-dimensional $\Gamma$-graded algebra. Now let

$$
a=\overline{\exp }^{g r}(A)=\limsup _{n \rightarrow \infty} \sqrt[n]{c_{n}^{g r}(A)} .
$$

The next lemma is the main step of the proof of Theorem 2 .

Lemma 7. Let $\Gamma$ be a commutative semigroup and let a in (16) be strictly greater than 1. If $A$ is graded simple then for any $\varepsilon>0$ and any $\delta>0$ there exists an increasing sequence of positive integers $n^{(1)}, n^{(2)}, \ldots$ such that

(i) $\sqrt[n]{c_{n}^{g r}(A)}>(1+\delta)(1-\varepsilon)$ for all $n=n^{(1)}, n^{(2)}, \ldots$; and

(ii) $n^{(q+1)}, n^{(q)} \leq d$, for all $q=1,2, \ldots$.

Proof. Clearly, there exists an integer $n^{(1)}$ such that

$$
c_{n^{(1)}}^{g r}(A)>(a-\varepsilon)^{n^{(1)}}
$$

and $n^{(1)}$ can be chosen arbitrary large. There are also $n_{1}, \ldots, n_{k} \geq 0$ such that $n_{1}+\cdots+n_{k}=n^{(1)}$ and

$$
\left(\begin{array}{c}
n^{(1)} \\
n_{1}, \ldots, n_{k}
\end{array}\right) c_{n_{1}, \ldots, n_{k}}(A)>\frac{1}{\left(n^{(1)}+1\right)^{k}}(a-\varepsilon)^{n^{(1)}} .
$$

(see (2)). Without loss of generality, we can suppose that $k=\mid$ Supp $A \mid$. Consider the $H=S_{n_{1}} \times \cdots \times S_{n_{k}}$-action on $P_{n_{1}, \ldots, n_{k}}$. It follows from (6), (7), (10) that there exist partitions $\lambda^{(1)} \vdash n_{1}, \ldots, \lambda^{(k)} \vdash n_{k}$ such that

$$
d_{\lambda^{(1)}} \cdots d_{\lambda^{(k)}}>\frac{1}{l_{n^{(1)}}^{g r}(A)} c_{n_{1}, \ldots, n_{k}}(A) .
$$

By Theorem 1 we have

$$
l_{n^{(1)}}^{g r}(A)<d\left(n^{(1)}+1\right)^{k(d+1)^{2}}
$$

hence

$$
d_{\lambda^{(1)}} \cdots d_{\lambda^{(k)}}>\frac{1}{d\left(n^{(1)}+1\right)^{k(d+1)^{2}}} c_{n_{1}, \ldots, n_{k}}(A) .
$$

and

$$
\left(\begin{array}{c}
n^{(1)} \\
n_{1}, \ldots, n_{k}
\end{array}\right) d_{\lambda^{(1)}} \cdots d_{\lambda^{(k)}}>\frac{1}{d\left(n^{(1)}+1\right)^{k(d+1)^{2}+k}}(a-\varepsilon)^{n^{(1)}} .
$$

There exists a multilinear polynomial

$$
f=f\left(x_{1}^{(1)}, \ldots, x_{n_{1}}^{(1)}, \ldots, x_{1}^{(k)}, \ldots, x_{n_{k}}^{(k)}\right) \notin I d^{g r}(A)
$$

where $x_{j}^{(i)} \in X_{g_{i}}$ for all $1 \leq i \leq k, 1 \leq j \leq n_{k}$ such that $f$ generates an irreducible $F[H]$-module with the character

$$
\chi_{H}(F[H] f)=\chi_{\lambda(1), \ldots, \lambda(k)} .
$$

There are homogeneous in $\Gamma$-grading $a_{1}^{1}, \ldots, a_{n_{1}}^{1}, \ldots, a_{1}^{k}, \ldots, a_{n_{k}}^{k}$ with $\operatorname{deg}_{\Gamma} a_{j}^{i}=$ $g_{i}$ such that

$$
Q=f\left(a_{1}^{1}, \ldots, a_{n_{k}}^{k}\right) \neq 0 .
$$


Since $\Gamma$ is associative and commutative it follows that $Q$ is homogeneous in $\Gamma$ grading of $A$. Therefore one can find $d^{\prime} \leq d$ and homogeneous $c_{1}, \ldots, c_{d^{\prime}} \in A$ satisfying the inequality

$$
\left(Q * c_{1} * \ldots * c_{d^{\prime}}\right) * Q \neq 0
$$

where $a * b$ denotes the right or the left multiplication by $b$ (otherwise $\operatorname{Span}<Q>$ is a graded ideal of $A$ ). Denote

$$
g_{1}=f_{1}=f\left(x_{1,1}^{(1)}, \ldots, x_{1, n_{1}}^{(1)}, \ldots, x_{1,1}^{(k)}, \ldots, x_{1, n_{k}}^{(k)}\right),
$$

where $x_{\alpha, \beta}^{(i)}$ are new homogeneous variables, $\operatorname{deg}_{\Gamma} x_{\alpha, \beta}^{(i)}=g_{i}$, and take

$$
g_{2}=\left(f_{1} * z_{1} * \cdots * z_{d^{\prime}}\right) * f_{2},
$$

where $\operatorname{deg}_{\Gamma} z_{1}=\operatorname{deg}_{\Gamma} c_{1}, \ldots, \operatorname{deg}_{\Gamma} z_{d^{\prime}}=\operatorname{deg}_{\Gamma} c_{d^{\prime}}$,

$$
f_{2}=f\left(x_{2,1}^{(1)}, \ldots, x_{2, n_{1}}^{(1)}, \ldots, x_{2,1}^{(k)}, \ldots, x_{2, n_{k}}^{(k)}\right),
$$

with $x_{\alpha, \beta}^{(i)} \in X_{g_{i}}$. Then $g_{2} \in P_{2 n_{1}+q_{1}, \ldots, 2 n_{k}+q_{k}}$ is not an identity of $A$, as follows from (18), and $q_{1}, \ldots, q_{k} \geq 0, q_{1}+\cdots+q_{k}=d^{\prime}$.

The square $H \times H$ of group $H$ acts on $\left\{x_{\alpha, \beta}^{(i)}\right\}$ where the first copy of $H$ acts on $\left\{x_{1, \beta}^{(i)}\right\}$, while the second copy acts on $\left\{x_{2, \beta}^{(i)}\right\}$ and

$$
\chi_{H \times H}\left(F[H \times H] g_{2}\right)=\chi_{\lambda^{(1)}, \ldots, \lambda(k)} \otimes \chi_{\lambda^{(1)}, \ldots, \lambda(k)} .
$$

Denote $n^{(2)}=2 n^{(1)}+d^{\prime}$. Repeating this procedure we construct for all $q=$ $3,4, \ldots$ a multilinear polynomial

$$
g_{q}=g_{q}\left(x_{1,1}^{(1)}, \ldots, x_{1, n_{1}}^{(1)}, \ldots, x_{q, 1}^{(k)}, \ldots, x_{q, n_{k}}^{(k)}, z_{1}, z_{2}, \ldots\right)
$$

of degre $n^{(q)}$ such that:

(i) all $x_{\alpha, \beta}^{(i)}, z_{j}$ are homogeneous and $x_{\alpha, \beta}^{(i)} \in X_{g_{i}}$;

(ii) $g_{q}$ is not an identity of $A$;

(iii) $n^{(q)}=q n^{(1)}+d^{(q)}, d^{(q)} \leq(q-1) d \leq d q, n^{(q)}-n^{(q-1)} \leq d$; and

(iv) $q$ copies $H^{q}=H \times \cdots \times H$ of $H$ acts on $g_{q}$ permuting $x_{\alpha, \beta}^{(i)}$ and $g_{q}$ generates an irreducible $F\left[H^{q}\right]$-module $M$ with

$$
\chi(M)=\left(\chi_{\lambda(1), \ldots, \lambda(k)}\right)^{\otimes q} .
$$

Denote $H(q)=S_{q n_{1}} \times \cdots \times S_{q n_{k}}$. Given $1 \leq i \leq k$, group $S_{q n_{i}}$ acts on $\left\{x_{1,1}^{(i)}, \ldots, x_{q, n_{i}}^{(i)}\right\}$. We can induce the $H^{q}$-action on $M$ to the $H(q)$-action. Consider the decomposition of $\widetilde{M}=F[H(q)] g_{q}$ into irreducible components,

$$
\chi_{H}(q)(\widetilde{M})=\sum_{\rho^{(1)} \vdash q n_{1}, \ldots, \rho^{(k)} \vdash q n_{k}} t_{\rho^{(1)}, \ldots, \rho^{(k)}} \chi_{\rho^{(1)}, \ldots, \rho^{(k)}} .
$$

It follows by the Richardson-Littlewood rule that for any $1 \leq i \leq k$, either $\rho^{(i)}=$ $q \lambda^{(i)}$ or $\rho^{(i)}$ is obtained from $\lambda^{(i)}$ by putting down one or more boxes of $D_{q \lambda^{(i)}}$. Then by Lemma 2 we have $\Phi\left(\rho^{(i)}\right) \geq \Phi\left(q \lambda^{(i)}\right)=\Phi\left(\lambda^{(i)}\right)$. Now, Lemma 1 implies the inequality

$$
d_{\rho^{(1)}} \cdots d_{\rho^{(k)}}>\left(\frac{1}{q n^{(1)}}\right)^{k(d+1)^{2}} d_{q \lambda^{(1)}} \cdots d_{q \lambda^{(k)}} .
$$


Recall that $g_{q}$ is not an identity of $A$. Hence there exist integers $p_{q, 1}, \ldots, p_{q, k} \geq 0$ such that $p_{q, 1}+\cdots+p_{q, k}=d^{(k)}$ and

$$
c_{q n_{1}+p_{q, 1}, \ldots, q n_{k}+p_{q, k}}(A) \geq d_{\rho^{(1)}} \cdots d_{\rho^{(k)}} .
$$

In particular,

$$
c_{n^{(q)}}^{g r}(A) \geq\left(\begin{array}{c}
q n^{(1)} \\
q n_{1}, \ldots, q n_{k}
\end{array}\right) d_{\rho^{(1)}} \cdots d_{\rho^{(k)}} .
$$

Note that for any partition $\mu \vdash m, \nu \vdash m$ with $\Phi(\mu) \geq \Phi(\nu)$ it follows by Lemma 1 that

$$
d_{\mu} \geq \frac{1}{m^{d^{2}+d}} \Phi(\mu)^{m} \geq \frac{1}{m^{d^{2}+d}} \Phi(\nu)^{m} \geq \frac{1}{m^{d^{2}+d+1}} d_{\nu} .
$$

Then by Lemma 6 and (19), the right hand side of (20) is not less than

$$
\left(\frac{1}{q n^{(1)}}\right)^{2 k(d+1)^{2}}\left(\frac{1}{n^{(1)}}\right)^{2 k q}\left[\left(\begin{array}{c}
n^{(1)} \\
n_{1}, \ldots, n_{k}
\end{array}\right) d_{\lambda^{(1)}} \cdots d_{\lambda^{(k)}}\right]^{q} .
$$

Now, (17) implies the following inequality

$$
c_{n^{(q)}}^{g r}(A) \geq\left(\frac{1}{q n^{(1)}}\right)^{2 k(d+1)^{2}}\left(\frac{1}{n^{(1)}}\right)^{2 k q}\left(\frac{1}{d\left(n^{(1)}+1\right)}\right)^{2 k(d+1)^{2} q}(a-\varepsilon)^{n^{(q)}} .
$$

Since $a>1$, by the assumptions of the lemma we then have

$$
(a-\varepsilon)^{q n^{(1)}} \geq \frac{(a-\varepsilon)^{n^{(q)}}}{a^{q d}}
$$

for all small enough $\varepsilon$. Hence

$$
\sqrt[n]{c_{n}^{g r}(A)}>D(a-\varepsilon),
$$

where $D=D_{1} D_{2}$,

$$
D_{1}=\left(\frac{1}{n^{(q)}}\right)^{\frac{2 k(d+1)^{2}}{n^{(q)}}}, \quad D_{2}=\left(\frac{1}{n^{(1)}}\right)^{\frac{2 k}{n^{(1)}}}\left(\frac{1}{d\left(n^{(1)}+1\right)}\right)^{\frac{2 k(d+1)^{2}}{n^{(1)}}}\left(\frac{1}{a}\right)^{\frac{d}{n^{(1)}+d}} .
$$

For small $\delta_{1}, \delta_{2}>0$ one can choose $n^{(1)}$ such that $D_{1}>\left(1-\delta_{1}\right)$ and $D_{2}>\left(1-\delta_{2}\right)$ for all $n^{(q)}, q \geq 1$. Finally, we can take $\delta_{1}, \delta_{2}$ small enough and get the inequality

$$
\sqrt[n]{c_{n}^{g r}(A)}>(1-\delta)(a-\varepsilon),
$$

for all $n=n^{(q)}, q=1,2, \ldots$.

Remark 1. In the proof of the previous lemma we used associativity and commutativity of $\Gamma$ only for getting relation (18). In case of an arbitrary groupoid $\Gamma$ the element $Q$ in (18) can be non-homogeneous in $\Gamma$-grading and hence an ideal I generated by $Q$ in $A$ can be strictly less than $A$. But if $A$ is simple in a non-graded sense then $I=A$ and relation (18) and Lemma 7 hold.

For completing the proof of the main result we need the following remark. Denote by $A n n A$ the annihilator of $A$. 
Lemma 8. Let $A$ be a $\Gamma$-graded algebra with a finite support of order $k$. If Ann $A=$ 0 then

$$
c_{n+1}^{g r}(A)>\frac{1}{8 k n^{k}} c_{n}^{g r}(A)
$$

for all sufficiently large $n$.

Proof. Denote Supp $A=\left\{g_{1}, \ldots, g_{k}\right\}$. It follows from (2) that there exist $n_{1}, \ldots, n_{k} \geq$ $0, n_{1}+\cdots+n_{k}=n$ such that

$$
\frac{1}{2 n^{k}} c_{n}^{g r}(A)<\frac{1}{(n+1)^{k}} c_{n}^{g r}(A)<c_{n_{1}, \ldots, n_{k}}(A) .
$$

Recall that $c_{n_{1}, \ldots, n_{k}}(A)=\operatorname{dim} P_{n_{1}, \ldots, n_{k}}(A)$ (see 4). Denote by $U_{i}, 1 \leq i \leq k$, the subspace of polynomials $f$ in $P_{n_{1}, \ldots, n_{k}}(A)$ such that $\varphi(f) A_{g_{i}}=0$ for all graded evaluations $\varphi: F\{X\} \rightarrow A$. Similarly, let $W_{i}, 1 \leq i \leq k$, be the subspace of polynomials $h \in P_{n_{1}, \ldots, n_{k}}(A)$ satisfying $A_{g_{i}} \varphi(h)=0$ for all graded evaluations $\varphi: F\{X\} \rightarrow A$. Denote

$$
V=U_{1} \cap \ldots \cap U_{k} \cap W_{1} \cap \ldots \cap W_{k} .
$$

If $f \in V$, then all values of $f$ in $A$ lie in $A n n A=0$, that is $V=0$. Suppose that

$$
\operatorname{dim} U_{1}, \ldots, \operatorname{dim} U_{k}, \ldots, \operatorname{dim} W_{1}, \ldots, \operatorname{dim} W_{k}>\frac{4 k-1}{4 k} N
$$

where $N=c_{n_{1}, \ldots, n_{k}}(A)$. Then $\operatorname{dim} V>\left(2 k \cdot \frac{4 k-1}{4 k}-(2 k-1)\right) N=\frac{1}{2} N$, that is $V \neq 0$, a contradiction. It follows that $\operatorname{dim} U_{i}<\frac{4 k-1}{4 k} N$ or $\operatorname{dim} W_{i}<\frac{4 k-1}{4 k} N$ for at least one $i$. Let, for instance, $\operatorname{dim} U_{1}<\frac{4 k-1}{4 k} N$. Denote by $T$ the codimension of $U_{1}$ in $P_{n_{1}, \ldots, n_{k}}(A)$. Then

$$
T>\frac{1}{4 k} c_{n_{1}, \ldots, n_{k}}(A)>\frac{1}{8 k n^{k}} c_{n}^{g r}(A)
$$

as follows from (21). Now if $f_{1}, \ldots, f_{T}$ are linearly independent modulo $U_{1}$ elements from $P_{n_{1}, \ldots, n_{k}}(A)$ then $f_{1} z, \ldots, f_{T} z$ are linearly independent elements in $P_{n_{1}+1, n_{2}, \ldots, n_{k}}(A)$, provided that $z$ is a new homogeneous indeterminate, $\operatorname{deg}_{\Gamma} z=$ $g_{1}$. Hence

$$
c_{n+1}^{g r}(A) \geq c_{n_{1}+1, n_{2}, \ldots, n_{k}}(A) \geq T>\frac{1}{8 k n^{k}} c_{n}^{g r}(A),
$$

and we are done.

Now we are ready to prove the main result of this paper.

Theorem 2. Let $\Gamma$ be a commutative semigroup and let $A=\bigoplus_{g \in \Gamma} A_{g}$ be a finite dimensional $\Gamma$-graded algebra. If $A$ is graded simple then there exists the limit

$$
\exp ^{g r}(A)=\lim _{n \rightarrow \infty} \sqrt[n]{c_{n}^{g r}(A)}
$$

Proof. Denote

$$
a=\overline{\exp }^{g r}(A)=\limsup _{n \rightarrow \infty} \sqrt[n]{c_{n}^{g r}(A)} .
$$

If $a=0$ then $A$ is nilpotent and $\exp ^{g r}(A)=0$. If $A$ is not nilpotent then $a \geq 1$. In the case $a=1$ the lower limit of $\sqrt[n]{c_{n}^{g r}(A)}$ is also 1 and we are done.

Let now $a>1$. By Lemma 7 there exists a sequence $n^{(1)}<n^{(2)}<\ldots$ such that

$$
c_{n}^{g r} \geq(1-\delta)^{n}(a-\varepsilon)^{n}
$$


for all $n=n^{(i)}, i \geq 1$, and $\varepsilon, \delta>0$ can be choosen arbitrary small.

Now let $m=n^{(i)}, m^{\prime}=n^{(i+1)}$ and let $m<n<m^{\prime}$. Then $n=m+p, 1 \leq p<d$. By Lemma 8 we have

$c_{n}^{g r}(A)=c_{m+p}^{g r}(A)>\left(\frac{1}{(8 k(m+p))}\right)^{p}(1-\delta)^{n}(a-\varepsilon)^{m}>\left(\frac{1}{8 k n}\right)^{d} \frac{1}{(a-\varepsilon)^{d}}(1-\delta)^{n}(a-\varepsilon)^{n}$.

Clearly, inequality (22) also holds for all $n=n^{(1)}, n^{(2)}, \ldots$, and for all small $\varepsilon, \delta>0$. Hence

$$
\liminf _{n \rightarrow \infty} \sqrt[n]{c_{n}^{g r}(A)} \geq(1-\delta)(a-\varepsilon)
$$

Since $\varepsilon, \delta$ are arbitrary we can conclude that

$$
\underline{\exp }^{g r}(A)=\liminf _{n \rightarrow \infty} \sqrt[n]{c_{n}^{g r}(A)}=a
$$

and the proof of the theorem is completed.

Finally, note that associativity and commutativity of $\Gamma$ was used only in the proof of Lemma 7 (see Remark 1). Hence for an arbitrary groupoid $\Gamma$ we have obtained the following result.

Theorem 3. Let $A=\bigoplus_{g \in \Gamma} A_{g}$ be a finite dimensional algebra graded by a groupoid $\Gamma$. If $A$ is simple then there exists its graded PI-exponent

$$
\exp ^{g r}(A)=\lim _{n \rightarrow \infty} \sqrt[n]{c_{n}^{g r}(A)}
$$

Acknowledgements. This research was supported by the Slovenian Research Agency grants P1-0292-0101, J1-7025-0101, J1-6721-0101, and J1-5435-0101, and the Russian Foundation for Basic Research grant 16-01-00113a. We thank the referee for comments and suggestions.

\section{REFERENCES}

[1] Giambruno, A., Zaicev, M., On codimension growth of finitely generated associative algebras, Adv. Math. 140 (1998), no. 2, $145-155$.

[2] Giambruno, A., M. Zaicev, M., Exponential codimension growth of P.I. algebras: an exact estimate, Adv. Math. 142 (1999), $221-243$.

[3] Giambruno, A., Regev, A., Zaicev, M., On the codimension growth of finite-dimensional Lie algebras, J. Algebra 220 (1999), no. 2, 466 - 474.

[4] Giambruno, A., Regev, A., Zaicev, M., Simple and semisimple Lie algebras and codimension growth, Trans. Amer. Math. Soc. 352 (2000), no. 4, 1935 - 1946.

[5] Zaitsev, M. V., Integrality of exponents of growth of identities of finite-dimensional Lie algebras. (Russian), Izv. Ross. Akad. Nauk Ser. Mat. 66 (2002), 23 - 48; English translation in Izv. Math. 66 (2002), $463-487$.

[6] Giambruno, A., Zaicev, M., Multialternating Jordan polynomials and codimension growth of matrix algebras, J. Lin. Alg. Appl. 422 (2007), no. 2-3, $372-379$.

[7] Giambruno, A., Zaicev, M., Codimension growth of special simple Jordan algebras, Trans. Amer. Math. Soc. 362 (2010), no. 6, 3107 - 3123.

[8] Giambruno, A., Shestakov, I., Zaicev, M., Finite-dimensional non-associative algebras and codimension growth, Adv. Appl. Math. 47 (2011), 125 - 139.

[9] Giambruno, A., Mishchenko, S., Zaicev, M., Codimensions of algebras and growth functions, Adv. Math. 217 (2008), no. 3, 1027 - 1052. 
[10] Zaicev, M., On existence of PI-exponents of codimension growth, Electr. Res. Announc. Math. Sci. 21 (2014), 113 - 119.

[11] Giambruno, A., Zaicev, M., On codimension growth of finite dimensional Lie superalgebras, J. London Math. Soc. 95 (2012), $534-548$.

[12] Giambruno, A., La Mattina, D., Graded polynomial identities and codimensions: computing the exponential growth, Adv. Math. 225 (2010), no. 2, 859 - 881.

[13] Repovš, D., Zaicev, M., Graded PI-exponents of simple Lie superalgebras, Ark. Mat. 54 (2016), no. 1, 147-156.

[14] Repovš, D., Zaicev, M., Graded identities of some simple Lie superalgebras, Algebr. Represent. Theory 17 (2014), no. 5, $1401-1412$.

[15] Repovš, D., Zaicev, M., Graded codimensions of Lie superalgebra b(2), J. Algebra 422 (2015), $1-10$.

[16] Berele, A., Regev, A., Applications of hook Young diagrams to PI algebras, J. Algebra 82 (1983), no. 2, $559-567$.

[17] Giambruno, A., Mishchenko, S., Zaicev, M., Algebras with intermediate growth of the codimensions, Adv. Appl. Math. 37 (2006), no. 3, 360 - 377.

[18] Zaicev, M., Graded identities in finite-dimensional algebras of codimensions of identities in associative algebras, Vestn. Mosk. Univ., Ser. I 70 (2015), no. 5, 54-57, English transl. in Mosc. Univ. Math. Bull. 70 (2015), no. 5, 234-236.

[19] Giambruno, A., Zaicev, M., Polynomial Identities and Asymptotic Methods, Mathematical Surveys and Monographs, 122, Amer. Math. Soc., Providence, RI, 2005.

[20] Drensky, V., Free Algebras and PI-algebras. Graduate Course in Algebra. Springer-Verlag Singapore, Singapore, 2000.

[21] Bahturin, Yu. and Drensky, V., Graded polynomial identities of matrices, Linear Algebra Appl. 357 (2002), 15 - 34.

[22] James, J., Kerber. A., The Representation Theory of the Symmetric Group, Encyclopedia of Mathematics and its Applications, 16, Addison-Wesley, London, 1981.

[23] Zaitsev, M., Repovš, D., Four dimensional simple algebra with a fractional PI-exponent, Mat. Zam. 95 (2014), no. 4, 538 - 553, English transl. in Math. Notes 95 (2014), no.4, 487 - 499.

D.D. Repovš, Faculty of Education, and Faculty of Mathematics and Physics, UniverSity of Ljubljana \& Institute of Mathematics, Physics and Mechanics, 1000 Ljubljana, SLOVENIA

E-mail address: dusan.repovs@guest.arnes.si

M.V. Zaicev, Department of Algebra, Faculty of Mathematics and Mechanics, Moscow State University, 119992 Moscow, Russia

E-mail address: zaicevmv@mail.ru 